\title{
Investigational Product Randomization Documentation
}

National Cancer Institute

\section{Source}

National Cancer Institute. Investigational Product Randomization Documentation. NCI

Thesaurus. Code C115707.

Records pertaining to the blinding and randomization of an investigational product (IP) and supplies. 